\title{
The real-space renormalization group and generating function for Penrose lattices
}

\author{
I Q You† and Franco Nori \\ Physics Department, The University of Michigan, Ann Arbor, MI 48109-1120, USA
}

Received 5 July 1993

\begin{abstract}
We present a real-space renormalization group ( $R G$ ) approach to study the electronic properties of the two-dimensional Penrose tilings. This approach is based on a recursive evaluation of the generating function which gives the density of states (DOS) of the system. The RG equations are derived in terms of the inflation rules of the Penrose tilings and the calculated Dos matches the results derived by purely numerical methods.
\end{abstract}

Real-space renormalization group (RG) approaches have been successful in describing the electronic properties of one-dimensional (1D) quasiperiodic systems [1]. However, it is a difficult problem to apply RG techniques to 2D problems [2]. Recently, Sire and Bellissard [3] developed an RG method by using the Schure formula to study a Laplace-like model on an octagonal quasiperiodic tiling. Their numerical calculations show that, in the low-t limit, the spectrum has a Cantor set structure. This result is consistent with those obtained for the periodic approximants of the octagonal lattice [4]. For the 2D Penrose tilings, You et al [5] developed a real-space RG scheme to calculate the local Green's functions for electrons in these quasiperiodic systems. Because of the limitation of this scheme, it only gives the local densities of states (DOSs) at several special sites and cannot be used to obtain the global DOS of the system. In this paper, we present a new RG approach to study the electronic properties of the $2 \mathrm{D}$ Penrose tilings. This approach is based on a recursive evaluation of the generating function [6,7] which involves the Hamiltonian of the system and gives the global Dos. It is important to point out that it is physically relevant to investigate the $2 \mathrm{D}$ one-electron tight-binding Hamiltonians, since they are directly related to experimental measurements of the critical temperature on 2D superconducting micronetworks [8].

Let us consider the following one-electron tight-binding Hamiltonian:

$$
H=\sum_{i}|i\rangle \epsilon_{i}\left\langle i\left|+\sum_{i, j}\right| i\right\rangle V_{i j}\langle j|
$$

where $|i\rangle$ is the Wannier state associated with the $i$ th vertex of a Penrose tiling, $\epsilon_{i}$ is the on-site potential at vertex $i$, and $V_{i j}$ the hopping integral between vertices $i$ and $j$. The generating function [6] is defined by

$$
\mathcal{G}(E)=\frac{1}{N} \ln \left[\int_{-\infty}^{+\infty}\left(\prod_{i=1}^{N} \mathrm{~d} u_{i}\right) \exp \left(-\frac{1}{2} U^{\mathrm{T}} \mathbf{X} U\right)\right]
$$

Here $U=\left(u_{1}, u_{2}, \ldots, u_{N}\right)$ is a column vector, $N$ is the number of sites in the system, and the matrix $X$ is defined by $X=Z \mathbf{l}-\mathrm{H}$, in which $Z=E+\mathrm{i} \eta, \mathbf{l}$ is the identity matrix

$\dagger$ On leave from the Laboratory of Modern Physics, Institute of Science and Technology, Xiangtan University, Xiangtan 411 105, Hunan, People's Republic of China. 
and $\boldsymbol{H}$ the $N \times N$ symmetric Hamiltonian matrix. Changing the integration variables to $V=T U$ where $T$ is the unimodular orthogonal transformation that diagonalizes $Z I-H$, the electronic density of states (DOS) can be derived [6]

$$
\rho(E)=\frac{2}{\pi} \lim _{\eta \rightarrow 0} \operatorname{Im} \frac{\partial \mathcal{G}}{\partial E}
$$

where Im denotes the imaginary part of a complex quantity. In principle, the limit $\eta \rightarrow 0$ in (3) must be taken; in practice, calculations are done with a finite but very small $\eta$. This means that there is a Lorentzian around each energy level instead of a $\delta$ function [6]. Here, by means of the RG technique, we calculate the generating function recursively by successively performing a fraction of the integrals in (2).

There are two kinds of Penrose lattices: one is constituted by thick and thin rhombuses $(R)$ and the other by kite- $(K)$ and dart-shaped $(D)$ tiles, and each lattice is obtained by inflating the other one. We distinguish these two kinds of lattices by denoting them as $R$ and KD Penrose tilings, respectively. In the $\mathrm{R}$ Penrose tiling, there exist eight types of vertices: $S, S 5, J, Q, K, S 3, S 4$, and $D[9]$, whose frequencies are $\tau^{-5} / \sqrt{5}, \tau^{-7} / \sqrt{5}, \tau^{-3}$, $\tau^{-4}, \tau^{-5}, \tau^{-6}, \tau^{-7}$, and $\tau^{-2}$, respectively [10], where $\tau$ is the golden mean $\tau=(\sqrt{5}+1) / 2$. In the KD Penrose tiling, there are seven types of vertices: $V I, I=1$ to 7 [11]. Since the $\mathrm{R}$ and KD Penrose tilings can be mapped to each other by inflation-rule transformations, one can derive, in terms of the vertex frequencies of the $R$ Penrose tiling, that the frequencies of the vertices $V I, I=1$ to 7 , are $\tau^{-4} / \sqrt{5}, \tau^{-6} / \sqrt{5}, \tau^{-2}, \tau^{-3}, \tau^{-4}, \tau^{-5}$, and $\tau^{-6}$, respectively. For the $R$ Penrose tiling, we denote by $H_{1}^{R}$ and $H_{2}^{R}$ the Hamiltonian matrices corresponding to the vertices of type $D$ and the remaining ones, respectively. $U^{\mathrm{T}} \mathbf{X}_{\mathrm{R}} U$ can be rewritten as

$$
U^{\mathrm{\top}} \mathbf{X}_{\mathrm{R}} U=U_{1}^{\mathrm{T}} \mathbf{X}_{11}^{\mathrm{R}} U_{1}+U_{1}^{\mathrm{T}} \mathbf{X}_{12}^{\mathrm{R}} U_{2}+U_{2}^{\mathrm{T}} \mathbf{X}_{21}^{\mathrm{R}} U_{1}+U_{2}^{\mathrm{T}} \mathbf{X}_{22}^{\mathrm{R}} U_{2},
$$

where $\mathbf{X}_{i i}^{\mathrm{R}}=\mathbf{Z l}-\mathbf{H}_{i}^{\mathrm{R}}, i=1,2$, and $\mathbf{X}_{12}^{\mathrm{R}}=\left(\mathbf{X}_{21}^{\mathrm{R}}\right)^{\mathrm{T}}$ represents the 'interactions' between the $D$-type vertices and the remaining ones.

When the D-type vertices are decimated, the R Penrose tiling becomes the KD Penrose tiling. Following the general procedure described in [6], we integrate over the variables associated with the $D$-type vertices to obtain

$$
\mathcal{G}_{\mathrm{R}}(E)=C_{\mathrm{KD}}^{\prime}+\mathcal{G}_{\mathrm{KD}}^{\prime}(E)
$$

Here $C_{\mathrm{KD}}^{\prime}$ is a constant which obeys the recursion relation

$$
C_{\mathrm{KD}}^{\prime}=C_{\mathrm{R}}+\frac{N_{D} \ln 2 \pi}{2 N}-\frac{1}{2 N} \ln \left[\operatorname{det}\left(\mathbf{X}_{11}^{\mathrm{R}}\right)\right]
$$

where $N_{D}$ is the number of $D$-type vertices and det denotes the determinant of a matrix; $\mathcal{G}_{\mathrm{KD}}^{\prime}(E)$ is the generating function of the scaled KD Penrose tiling associated with

$$
\mathbf{X}_{\mathrm{KD}}^{\prime}=\mathbf{X}_{22}^{\mathrm{R}}-\mathbf{X}_{21}^{\mathrm{R}}\left(\mathbf{X}_{11}^{\mathrm{R}}\right)^{-1} \mathbf{X}_{12}^{\mathrm{R}}
$$

where $X_{\mathrm{KD}}^{\prime}=\mathrm{ZI}-\mathrm{H}_{\mathrm{KD}}^{\prime}$ determines the renormalized Hamiltonian of the scaled $\mathrm{KD}$ Penrose tiling. Analogously, the KD Penrose tiling is converted to the $\mathrm{R}$ Penrose tiling if the vertices of type $V 3$ are eliminated and one obtains

$$
\mathcal{G}_{\mathrm{KD}}(E)=C_{\mathrm{R}}^{\prime}+\mathcal{G}_{\mathrm{R}}^{\prime}(E)
$$

where $C_{\mathrm{R}}^{\gamma}$ satisfies the recursion relation

$$
C_{\mathrm{R}}^{\prime}=C_{\mathrm{KD}}+\frac{N_{V^{3}} \ln 2 \pi}{2 N}-\frac{1}{2 N} \ln \left[\operatorname{det}\left(\mathbf{X}_{11}^{\mathrm{KD}}\right)\right]
$$


in which $N_{V 3}$ is the number of V3-type vertices, and $\mathcal{G}_{\mathrm{R}}^{\prime}(E)$ is the generating function of the scaled $\mathrm{R}$ Penrose tiling with

$$
\mathbf{X}_{\mathrm{R}}^{\prime}=\mathbf{X}_{22}^{\mathrm{KD}}-\mathbf{X}_{21}^{\mathrm{KD}}\left(\mathbf{X}_{11}^{\mathrm{KD}}\right)^{-1} \mathbf{X}_{12}^{\mathrm{KD}}
$$

which gives the renormalized Hamiltonian of the scaled $\mathrm{R}$ Penrose tiling. In principle, an $\mathrm{R}$ or $\mathrm{KD}$ Penrose tiling can be directly inflated to the same kind of Penrose tiling, but one needs to decimate simultaneously more than one type of vertex. This makes the problem very complicated because many interactions are involved among decimated vertices and between the decimated and undecimated vertices.

By infinite iterations of (5) and (8), the generating function of a Penrose tiling is obtained and thus, according to (3), the DOS of the quasiperiodic system is determined. However, starting from a Harniltonian with finite number of parameters, when the Penrose tiling is inflated, one obtains a renormalized Hamiltonian which depends on an increasing number of parameters at each decimating step. This is, of course, the case when RG techniques are applied to 2D problems either magnetic or electronic. Similar problems have also been found on the octagonal tiling [3] by employing RG techniques. Therefore, in practice, one can only solve the electronic problem of the Penrose tiling in an approximation fashion. For the $\mathrm{R}$ and KD Penrose tilings, we choose the parameters of the Hamiltonian as follows. The on-site potentials are assigned to be $E_{i}, i=1$ to 8 , for the eight types of vertices in the R Penrose tiling: $S, S 5, J, Q, K, S 3, S 4$, and $D$; and $\varepsilon_{i}, i=1$ to 7 , for the seven types of vertices in the $\mathrm{KD}$ Penrose tiling: $V I, I=1$ to 7 . For the bonds associated with the edges of the tiles in the $\mathrm{R}$ and $\mathrm{KD}$ Penrose tilings, there exist five Iocal configurations of bonds in each Penrose tiling. We choose the hopping integrals $V_{i j}$ to be non-zero for these bonds and, in addition, for the bonds associated with some of the diagonals of the tiles. The bonds with non-zero hopping integrals are shown in figures 1(a) and (b). In our RG approach, the truncating approximation is used, i.e. we truncate the renormalized Hamiltonian to keep both the number and the type of the parameters to be the same as the original Hamiltonian. In terms of $(7)$ and $(10),[6,7]$ when the truncating approximation is employed, we obtain the following two RG equations:

$$
\begin{array}{llll}
\varepsilon_{1}^{\prime}=E_{1}+5 t_{3}^{2} \Omega & \varepsilon_{2}^{\prime}=E_{2} & \varepsilon_{3}^{\prime}=E_{3}+2 t_{2}^{2} \Omega & \varepsilon_{4}^{\prime}=E_{4}+2 t_{6}^{2} \Omega \\
\varepsilon_{5}^{\prime}=E_{5}+\left(2 t_{3}^{2}+t_{6}^{2}\right) \Omega & \varepsilon_{6}^{\prime}=E_{6}+4 t_{2}^{2} \Omega & \varepsilon_{7}^{\prime}=E_{7}+2 t_{2}^{2} \Omega & \\
k_{1}^{\prime}=t_{5}+2 t_{2} t_{6} \Omega & k_{2}^{\prime}=t_{4}+t_{2} t_{6} \Omega & k_{3}^{\prime}=t_{8}+2 t_{2} t_{3} \Omega & k_{4}^{\prime}=t_{8}+t_{2} t_{3} \Omega \\
k_{5}^{\prime}=t_{8} & k_{6}^{\prime}=t_{1} & k_{7}^{\prime}=t_{2}^{2} \Omega & k_{8}^{\prime}=t_{3} t_{6} \Omega
\end{array}
$$

where $\Omega=1 /\left(Z-E_{8}\right)$, and

$$
\begin{array}{llll}
E_{1}^{\prime}=\varepsilon_{2}+5 k_{6}^{2} \theta & E_{2}^{\prime}=\varepsilon_{1}+5 k_{3}^{2} \theta & E_{3}^{\prime}=\varepsilon_{5}+\left(2 k_{2}^{2}+k_{3}^{2}\right) \theta & E_{4}^{\prime}=\varepsilon_{6}+k_{6}^{2} \theta \\
E_{5}^{\prime}=\varepsilon_{7}+3 k_{6}^{2} \theta & E_{6}^{\prime}=\varepsilon_{1}+3 k_{3}^{2} \theta & E_{7}^{\prime}=\varepsilon_{1}+4 k_{3}^{2} \theta & E_{8}^{\prime}=\varepsilon_{4}+2 k_{2}^{2} \theta \\
t_{1}^{\prime}=k_{8}+2 k_{2} k_{3} \theta & t_{2}^{\prime}=k_{8}+k_{2} k_{3} \theta & t_{3}^{\prime}=k_{5}+2 k_{2} k_{6} \theta & t_{4}^{\prime}=k_{4}+k_{2} k_{6} \theta \\
t_{5}^{\prime}=k_{3} & t_{6}^{\prime}=k_{1} & t_{7}^{\prime}=k_{2}^{2} \theta & t_{8}^{\prime}=k_{3} k_{6} \theta
\end{array}
$$

with $\theta=1 /\left(Z-\varepsilon_{3}\right)$. The error introduced in the truncating approximation is small for high orders, and do not significantly influence the resulting spectra.

To obtain the generating function, we need to evaluate the quantities $\ln \left[\operatorname{det}\left(\mathbf{X}_{11}^{R}\right)\right]$ in (6) and $\ln \left[\operatorname{det}\left(\mathbf{X}_{11}^{\mathrm{KD}}\right)\right]$ in (9) at each decimating step.

The quantity $\operatorname{det}\left(X_{11}^{R}\right)$ is equal to the product of the eigenvalues of $\mathrm{ZI}-\mathbf{H}_{1}^{\mathrm{R}}$, where $\mathrm{H}_{1}^{\mathrm{R}}$ is the Hamiltonian associated with the vertices of type $D$; while $\operatorname{det}\left(\mathbf{X}_{11}^{\mathrm{KD}}\right)$ is the product of the eigenvalues of $\mathbf{Z I}-\mathbf{H}_{1}^{K D}$, where $\mathrm{H}_{1}^{\mathrm{KD}}$ is the Hamiltonian associated with the vertices of type V3. For the $D$-type vertices, only two kinds of local configurations are formed; one 

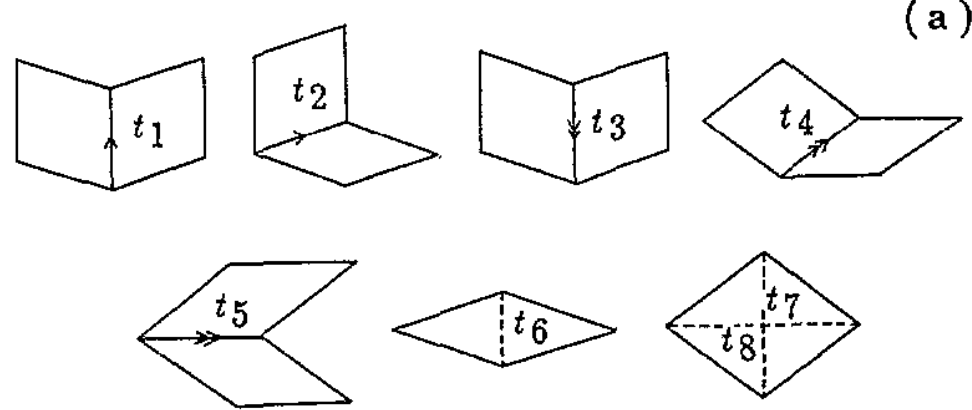

(b)
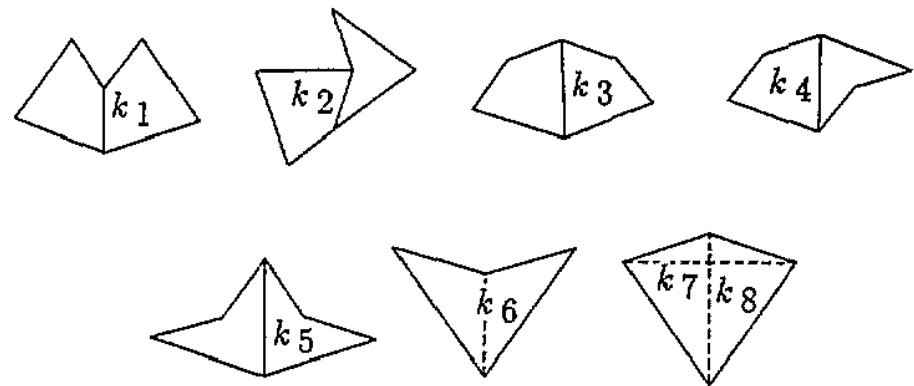

Figure 1. Assigned hopping integrals for (a) $\mathrm{R}$ and (b) $\mathrm{KD}$ Penrose tilings.

(a)
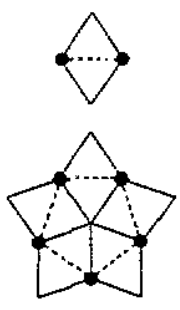

Figure 2. (a) Two kinds of local configurations formed by $D$-type vertices; (b) four kinds of local configurations constituted by V 3 -type vertices. (b)
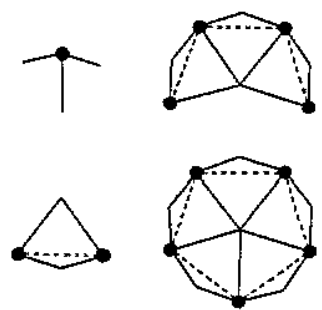

is the two-vertex chain and the other is the five-vertex ring (see figure 2(a)). In each of these configurations, adjoining $D$-type vertices are connected by the bond $t 7$. In terms of the frequencies of the vertices in the $R$ Penrose tiling, we obtain that the numbers of these two kinds of local configurations are $(\sqrt{5}-2) \tau^{-2} N_{R}$ and $(5-2 \sqrt{5}) \tau^{-2} N_{R} / 5$, respectively, where $N_{R}$ is the vertex number of the $R$ Penrose tiling. Since these local configurations are not connected with one another, thus $\ln \left[\operatorname{det}\left(\mathbf{X}_{11}^{\mathrm{R}}\right)\right]$ is given by

$$
\begin{aligned}
\ln \left[\operatorname{det}\left(X_{11}^{\mathrm{R}}\right)\right]= & \tau^{-2} N_{\mathrm{R}}\left\{(\sqrt{5}-2)\left[\ln \left(Z-E_{8}-t_{7}\right)+\ln \left(Z-E_{8}+t_{7}\right)\right]\right. \\
& +\frac{5-2 \sqrt{5}}{5}\left[\ln \left(Z-E_{8}-2 t_{7}\right)+2 \ln \left(Z-E_{8}-\tau^{-1} t_{7}\right)\right. \\
& \left.\left.+2 \ln \left(Z-E_{8}+\tau t_{7}\right)\right]\right\} .
\end{aligned}
$$


The vertices of type $V 3$ in the $\mathrm{KD}$ Penrose tiling constitute four kinds of local configurations, which are isolated V3-type vertex, two-vertex chain, four-vertex chain and five-vertex ring, respectively (see figure $2(\mathrm{~b})$ ). It is derived that the numbers of these local configurations are successively $(2 \sqrt{5}-4) \tau^{-2} N_{\mathrm{KD}},(5 \sqrt{5}-11) \tau^{-2} N_{\mathrm{KD}} / 2,(9-4 \sqrt{5}) \tau^{-2} N_{\mathrm{KD}}$, and $(9 \sqrt{5}-20) \tau^{-2} N_{\mathrm{KD}} / 5$, where $N_{\mathrm{KD}}$ is the vertex number of the $\mathrm{KD}$ Penrose tiling. Note that no $V 3$ three-vertex chain exists, since in the standard $\mathrm{KD}$ Penrose tiling with only seven types of vertex $(V 1, V 2, \ldots, V 7)$ there are only four kind of local configurations considered by the V3-type vertices of the tiling. The quantity $\ln \left[\operatorname{det}\left(\mathbf{X}_{11}^{K D}\right)\right]$ is given by

$$
\begin{aligned}
\ln \left[\operatorname{det}\left(\mathbf{X}_{11}^{\mathrm{KD}}\right)\right]= & \tau^{-2} N_{\mathrm{KD}}\left\{(2 \sqrt{5}-4) \ln \left(Z-\varepsilon_{3}\right)+\frac{5 \sqrt{5}-11}{2}\left[\ln \left(Z-\varepsilon_{3}-k_{7}\right)\right.\right. \\
& \left.+\ln \left(Z-\varepsilon_{3}+k_{7}\right)\right]+(9-4 \sqrt{5})\left[\ln \left(Z-\varepsilon_{3}-\tau k_{7}\right)\right. \\
& +\ln \left(Z-\varepsilon_{3}+\tau k_{7}\right)+\ln \left(Z-\varepsilon_{3}-\tau^{-1} k_{7}\right) \\
& \left.+\ln \left(Z-\varepsilon_{3}+\tau^{-1} k_{7}\right)\right]+\frac{9 \sqrt{5}-20}{5}\left[\ln \left(Z-\varepsilon_{3}-2 k_{7}\right)\right. \\
& \left.\left.+2 \ln \left(Z-\varepsilon_{3}-\tau^{-1} k_{7}\right)+2 \ln \left(Z-\varepsilon_{3}+\tau k_{7}\right)\right]\right\} .
\end{aligned}
$$

In terms of the values of $\ln \left[\operatorname{det}\left(\mathbf{X}_{11}^{\mathrm{R}}\right)\right]$ and $\ln \left[\operatorname{det}\left(\mathbf{X}_{11}^{\mathrm{KD}}\right)\right]$ at different decimating steps, we finally obtain from (3), (5) and (8) the global Doss for the $\mathrm{R}$ and $\mathrm{KD}$ Penrose tilings

$$
\begin{aligned}
\rho_{\mathrm{R}}(E)=-\frac{1}{\pi} & \lim _{\eta \rightarrow 0} \operatorname{Im} \sum_{n=0}^{\infty}\left\{\frac{1}{\tau^{2 n+2}}\left[(\sqrt{5}-2) \sum_{i=1}^{2} \frac{Q_{i}^{(2 n)}}{P_{i}^{(2 n)}}+\frac{5-2 \sqrt{5}}{5} \sum_{i=3}^{5} \frac{Q_{i}^{(2 n)}}{P_{i}^{(2 n)}}\right]\right. \\
& +\frac{1}{\tau^{2 n+3}}\left[(2 \sqrt{5}-4) \frac{q_{1}^{(2 n+1)}}{p_{1}^{(2 n+1)}}+\frac{5 \sqrt{5}-11}{2} \sum_{i=2}^{3} \frac{q_{i}^{(2 n+1)}}{p_{i}^{(2 n+1)}}\right. \\
& \left.\left.+(9-4 \sqrt{5}) \sum_{i=4}^{7} \frac{q_{i}^{(2 n+1)}}{p_{i}^{(2 n+1)}}+\frac{9 \sqrt{5}-20}{5} \sum_{i=8}^{10} \frac{q_{i}^{(2 n+1)}}{p_{i}^{(2 n+1)}}\right]\right\}
\end{aligned}
$$

and

$$
\begin{aligned}
\rho_{\mathrm{KD}}(E)=-\frac{1}{\pi} & \lim _{\eta \rightarrow 0} \operatorname{Im} \sum_{n=0}^{\infty}\left\{\frac { 1 } { \tau ^ { 2 n + 2 } } \left[(2 \sqrt{5}-4) \frac{q_{1}^{(2 n)}}{p_{1}^{(2 n)}}+\frac{5 \sqrt{5}-11}{2} \sum_{i=2}^{3} \frac{q_{i}^{(2 n)}}{p_{i}^{(2 n)}}\right.\right. \\
& +\left(9-4 \sqrt{5)} \sum_{i=4}^{7} \frac{q_{i}^{(2 n)}}{p_{i}^{(2 n)}}+\frac{9 \sqrt{5}-20}{5} \sum_{i=8}^{10} \frac{q_{i}^{(2 n)}}{p_{i}^{(2 n)}}\right] \\
& \left.+\frac{1}{\tau^{2 n+3}}\left[(\sqrt{5}-2) \sum_{i=1}^{2} \frac{Q_{i}^{(2 n+1)}}{P_{i}^{(2 n+1)}}+\frac{5-2 \sqrt{5}}{5} \sum_{i=3}^{5} \frac{Q_{i}^{(2 n+1)}}{P_{i}^{(2 n+1)}}\right]\right\} .
\end{aligned}
$$

where

$$
\begin{array}{lll}
P_{1}^{(n)}=Z-E_{8}^{(n)}-t_{7}^{(n)} & P_{2}^{(n)}=Z-E_{8}^{(n)}+t_{7}^{(n)} & P_{3}^{(n)}=Z-E_{8}^{(n)}-2 t_{7}^{(n)} \\
P_{4}^{(n)}=Z-E_{8}^{(n)}-\tau^{-1} t_{7}^{(n)} & P_{5}^{(n)}=Z-E_{8}^{(n)}+\tau t_{7}^{(n)} & p_{1}^{(n)}=Z-\varepsilon_{3}^{(n)} \\
p_{2}^{(n)}=Z-\varepsilon_{3}^{(n)}-k_{7}^{(n)} & p_{3}^{(n)}=Z-\varepsilon_{3}^{(n)}+k_{7}^{(n)} & p_{4}^{(n)}=Z-\varepsilon_{3}^{(n)}-\tau k_{7}^{(n)} \\
p_{5}^{(n)}=Z-\varepsilon_{3}^{(n)}+\tau k_{7}^{(n)} & p_{6}^{(n)}=Z-\varepsilon_{3}^{(n)}-\tau^{-1} k_{7}^{(n)} & p_{7}^{(n)}=Z-\varepsilon_{3}^{(n)}+\tau^{-1} k_{7}^{(n)} \\
p_{8}^{(n)}=Z-\varepsilon_{3}^{(n)}-2 k_{7}^{(n)} & p_{9}^{(n)}=Z-\varepsilon_{3}^{(n)}-\tau^{-1} k_{7}^{(n)} & p_{10}^{(n)}=Z-\varepsilon_{3}^{(n)}+\tau k_{7}^{(n)}
\end{array}
$$


and

$$
\begin{array}{ll}
Q_{i}^{(n)}=\partial P_{i}^{(n)} / \partial E & i=1 \text { to } 3 \\
Q_{i}^{(n)}=2 \partial P_{i}^{(n)} / \partial E & i=45 \\
q_{i}^{(n)}=\partial p_{i}^{(n)} / \partial E & i=1 \text { to } 8 \\
q_{i}^{(n)}=2 \partial p_{i}^{(n)} / \partial E & i=910 .
\end{array}
$$

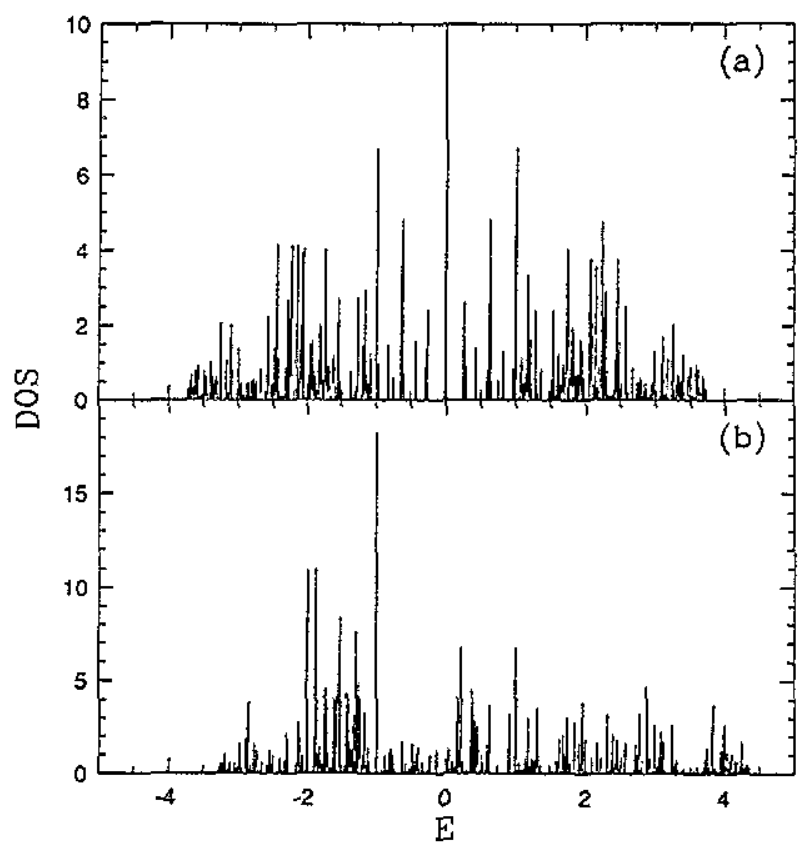

Figure 3. Dos (arbitrary units) of $\mathrm{R}$ Penrose tiling, in which the on-site potentials and hopping integrals are chosen to be (a) $E_{i}=0, i=1$ to 8 , and $t_{i}=1, i=1$ to $5, t_{6}=i_{7}=t_{8}=0$; (b) same as (a) except that $t_{6}=1$. In figures 3 and $4, \eta=0.001$.

Figure 3 presents the Doss of the R Penrose tiling. In figure 3(a) the on-site potentials and the hopping integrals are chosen to be $E_{i}=0, i=1$ to 8 , and $t_{i}=1, i=1$ to $5, t_{6}=t_{7}=t_{8}=0$, corresponding to the vertex model on the R Penrose tiling [12-14]. Kohmoto and Sutherland [12] numerically studied the electronic structure of the vertex model on the R Penrose tiling and found that: (1) in the DOS, there is a zero-width central peak at zero energy, on which a gap with finite size is centred; (2) the spectrum shows a complicated structure, particularly near the spectrum edges. The DOS shown in figure 3(a) exhibits these features. One can see that the Dos has hierarchical structure, and a very high and isolated peak appears at $E=0$, which is due to infinitely degenerate localized states in the R Penrose tiling. Arai et al [13] found six types of zero-energy localized states, of which two are ring states [12]. These states are termed confined states by them, because they are confined on certain local configurations. In the numerical calculations, Liu and $\mathrm{Ma}$ [14] found that when the bond $t_{6}$ is considered, which corresponds to the short diagonal of the thin rhombus, the calculated integrated Dos shows a very small jump at $E=0$. They suggested that the degeneracy of confined states are drastically reduced as the bond $t_{6}$ is included. Figure $3(\mathrm{~b})$ presents the DOS of the $\mathrm{R}$ Penrose tiling, for which $t_{6}=1$ and other parameters are chosen to be the same as those of the vertex model. It can be seen that the DOS shows hierarchical structure and the whole spectrum shifts rightwards. In this figure 
no isolated peak is observed exactly at $E=0$, but some low isolated peaks are found in the vicinity of the zero energy. The DOSs of the KD Penrose tiling are shown in figure 4. These Doss exhibit hierarchical structures, just as those of the $R$ Penrose tiling. For the vertex model, the DOS has a very high peak at $E=0$ (see figure 4(a)). This peak appears to be isolated but the gap centred on it is very narrow. When the bond associated with the short diagonal of the dart is included, the DOS shifts rightwards and no isolated peak occurs at $E=0$ (see figure $4(\mathrm{~b})$ ).

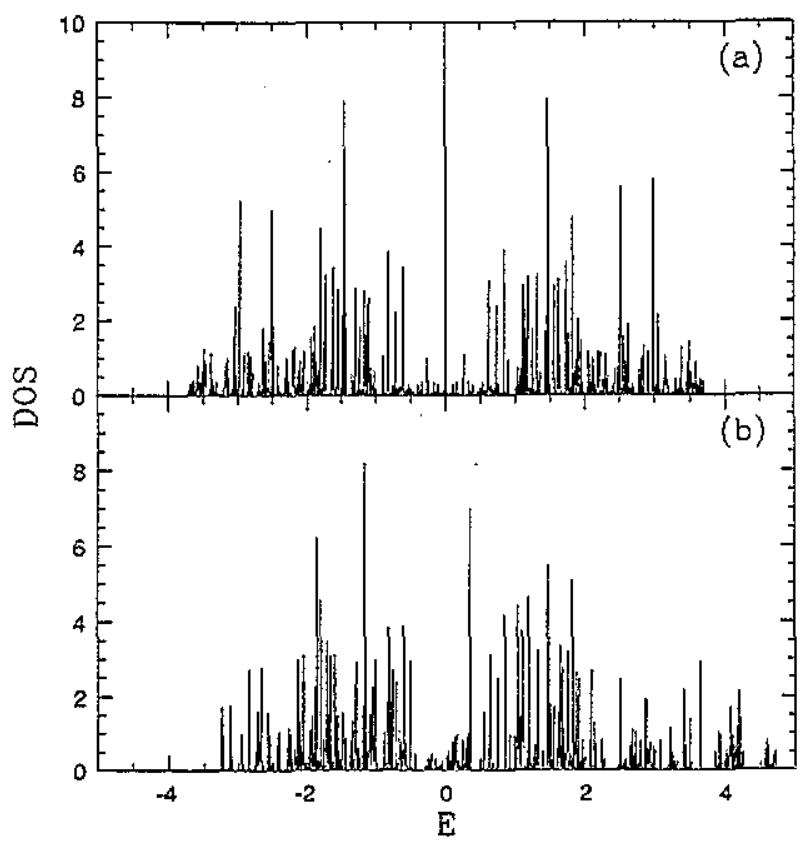

Figure 4. Dos (arbitrary units) of KD Penrose tiling, in which (a) $\varepsilon_{i}=0, i=1$ to 7 , and $k_{i}=1$, $i=1$ to $5, k_{6}=k_{7}=k_{8}=0$; (b) same as (a) except that $k_{6}=1$.

It is well known that for the 1D quasicrystalline case, the energy spectrum is a Cantor set of zero Lebesgue measure. For the energy spectra of a 2D Penrose tiling, the Lebesgue measure seems to be finite, since fewer gaps appear in the Dos. However, from our approach we cannot make rigorous statements about this. What about the behaviour of the singular points where the peaks are? How the peaks scale as a function of systems size? The DOS given in figures 3 and 4 are in fact average DOS, namely the DOS divided by the number, $N$, of sites in the system. As $N$ grows to infinity, the isolated peak at $E=0$ is constant in the average DOS. This implies that the degeneracy of the localized mode is proportional to $N$ for a very large system size. Finally, and as expected, the truncation of the hierarchy becomes more pronounced, and the RG approach gives better results, when the hopping constants are small (and not all the on-site energies are zero).

It was demonstrated above that the Doss of the vertex model on the $\mathrm{R}$ and $\mathrm{KD}$ Penrose tilings show the general features of the energy spectra of these quasiperiodic systems. However, because of the truncating approximation employed, the calculated Doss are narrower than the energy spectra obtained by others $[11,12]$. If this approximation is not used, the parameters of the Hamiltonian increase at each decimating step and the parameters corresponding to the locally equivalent vertices or bonds can now take several different values. When these parameters are averaged according to their local configurations, 
approximate RG equations associated with the inflation rules of the Penrose tilings can be also derived and it was found that the calculated Doss become broader.

In this article, the parameters of the Hamiltonian are chosen in such a way that their values are assigned to be equal for the locally equivalent vertices or bonds. If these vertices and bonds are further distinguished in wider regions, the initial Hamiltonian will have more parameters and the accuracy of the calculated DOS will improve. We are currently pursuing studies along this line.

We acknowledge conversations with $\mathrm{Q}$ Niu, and useful suggestions on the manuscript by one of the referees. This work was supported in part by NSF grant No DMR-90-01502 (FN) and the National Natural Science Foundation of China (JQY).

\section{References}

[1] Niu Q and Nori F 1986 Phys. Rev. Lett. 57 2057; 1990 Phys. Rev. B 4210329

[2] Burkhardt $\mathrm{T} W$ and van Leeuwen J M J (ed) 1982 Real-Space Renormalization (Berlin: Springer)

[3] Sire C and Bellissard J 1990 Europhys. Lett. 11439

[4] Benza V G and Sire C 1991 Phys. Rev. B 4410343

Passaro B, Sire C and Benza V G 1992 Phys. Rev. B 4613751

[5] You J Q, Yan J R, Zhong J X and Yan X H 1992 Europhys. Lett. 17231

[6] Lemieux M-A and Tremblay A-M S 1987 Phys. Rev. B 361463

[7] Ashraff J A and Stinchcombe R B 1989 Phys. Rev. B 392670

[8] Niu Q and Nori F 1989 Phys. Rev. B 392134

Nori F et al 1987 Phys. Rev. B 36 8338; 1988 Physica B 152105

[9] de Bruijn N G 1981 Ned. Akad. Weten. Proc. A 84 39, 53

[10] Henley C L 1986 Phys, Rev. B 34797

[11] Odagaki T and Nguyen D 1986 Phys. Rev. B 332184

[12] Kohmoto $M$ and Sutherland B 1986 Phys. Rev. Lett. 562740

[13] Arai M, Tokihiro T, Fujiwara T and Kohmoto M 1988 Phys. Rev. B 381621

[14] Liu Y and Ma P 1991 Phys. Rev. B 431378 